\title{
Effect of swirling device on flow behavior in a supersonic separator for natural gas dehydration
}

Wen, Chuang; Li, Anqi ; Walther, Jens Honore; Yang, Yan

\section{Published in:}

Separation and Purification Technology

Link to article, DOI:

10.1016/j.seppur.2016.05.019

Publication date:

2016

Document Version

Peer reviewed version

Link back to DTU Orbit

Citation (APA):

Wen, C., Li, A., Walther, J. H., \& Yang, Y. (2016). Effect of swirling device on flow behavior in a supersonic separator for natural gas dehydration. Separation and Purification Technology, 168, [68-73].

https://doi.org/10.1016/j.seppur.2016.05.019

\section{General rights}

Copyright and moral rights for the publications made accessible in the public portal are retained by the authors and/or other copyright owners and it is a condition of accessing publications that users recognise and abide by the legal requirements associated with these rights.

- Users may download and print one copy of any publication from the public portal for the purpose of private study or research.

- You may not further distribute the material or use it for any profit-making activity or commercial gain

- You may freely distribute the URL identifying the publication in the public portal 


\title{
Effect of swirling device on flow behavior in a supersonic
}

\section{separator for natural gas dehydration}

\author{
Chuang Wen ${ }^{\text {a,b }}$, Anqi Li ${ }^{a}$, Jens Honore Walther ${ }^{b, c}$, Yan Yang ${ }^{a, *}$ \\ ${ }^{a}$ Jiangsu Key Laboratory of Oil-Gas Storage and Transportation Technology, \\ Changzhou University, Changzhou, 213016, China \\ ${ }^{\mathrm{b}}$ Section of Fluid Mechanics, Coastal and Maritime Engineering, Department of \\ Mechanical Engineering, Technical University of Denmark, Nils Koppels Allé, 2800 \\ Kgs. Lyngby, Denmark \\ ${ }^{\mathrm{c}}$ Chair of Computational Science, ETH Zürich, Clausiusstrasse 33 ETH-Zentrum, CLT \\ F 11, CH-8092 Zürich, Switzerland \\ *Corresponding author, Tel: +86 5198329 0280; Fax: +86 51983295530. \\ E-mail address: yyan-petroleum@cczu.edu.cn (Y Yang)
}

\begin{abstract}
The supersonic separator is a revolutionary device to remove the condensable components from gas mixtures. One of the key issues for this novel technology is the complex supersonic swirling flow that is not well understood. A swirling device composed of an ellipsoid and several helical blades is designed for an annular supersonic separator. The supersonic swirling separation flow of natural gas is calculated using the Reynolds Stress model. The results show that the viscous heating and strong swirling flow cause the adverse pressure in the annular channel, which negatively affects the separation performance. When the swirling flow passes through the annular nozzle, it will damage the expansion characteristics of the annular nozzle. The blade angles and numbers are both optimized by evaluating the swirling and expansion effects for the supersonic separation.
\end{abstract}

Key words: gas separation; supersonic; swirling flow; helical blade

\section{Introduction}


Natural gas usually contains a certain amount of water vapor when it is extracted from the underground. When the gas reaches the wellhead, the water vapor in the natural gas will condense into the liquid water as a result of the reduction in temperature, which causes a range of hazards to the pipeline for natural gas storage and transportation. For example, it may result in the formation of the hydrates that may block the pipelines. The liquid water may also react with the acid gas, namely, carbon dioxide or hydrogen sulfide, to aggravate the corrosion problem. Therefore, the gas dehydration is an important part in natural gas processing treatment.

At present, the conventional techniques for natural gas dehydration mainly include the refrigeration, adsorption [1], absorption [2, 3], membrane separation [4, 5]. The supersonic swirling separation is a technical innovation in the field of natural gas dehydration $[6,7]$. In a supersonic separator, natural gas is accelerated to a supersonic velocity using the influence of a Laval nozzle, and correspondingly forms a low pressure and temperature condition, which results in the condensation of the water vapor and heavy hydrocarbon components. The swirling device generates a large centrifugal force that will eject the condensed droplets onto the walls, which will be discharged after they enter into the collection space. The dry gas resides at the center of the tube and flows out from the diffuser, in which the gas speed reduces to a subsonic as a result of the shock wave.

Jassim et al. [8, 9] studied the supersonic flow characteristics of natural gas in a Laval nozzle under high pressure using computational fluid dynamics (CFD) simulations, focusing on the effect of the nozzle structure on natural gas dynamic parameters. The influence of the operating parameters on the high speed flow characteristics of natural gas through a Laval nozzle was mathematically investigated by Karimi and Abdi [10]. Malyshkina [11, 12] took account of a strong swirl in the 
numerical simulation of a single gas field in a supersonic separator. Yang et al. [13, 14] performed detailed CFD simulations to study the pressure recovery characteristics and real gas effects based on a single gas flow without considering swirl. Vaziri \& Shahsavand [15] optimized the axial, radial and circumferential components of the inlet velocity by evaluating the swirl strength and centrifugal acceleration with the assumption of a single gas flow in a supersonic separator. The results showed that the increasing pressure and temperature decreased the swirl number and centrifugal acceleration. Ma et al. [16] developed a two-fluid model to simulate the condensation flow in a converging-diverging nozzle based on the ideal gas assumption. The CFD model was validated with experimental data. The mathematical model was used to calculate the nucleation and condensation process in a supersonic separator with a strong swirl flow [17]. Numerical simulations have been conducted on the condensation processes of the water vapor in the supersonic conditions by Shooshtari and Shahsavand [18, 19]. The effect of the nozzle geometry on the condensing parameters was analyzed with one-dimensional assumption. Castier [20] also carried out some numerical simulations of natural gas flow within a Laval nozzle both in consideration of the single flow and the phase equilibrium. Haghighi et al [21] addressed the recent developments of the supersonic separation technique, and pointed out that the swirl generation device was an important part of the design of these separators and further research could potentially increase the knowledge base to improve the swirl generation effectiveness significantly.

One of the complex issues for the supersonic separation technology is to employ the centrifugal force to remove the condensed droplets from the gas-liquid mixtures. Therefore, it is necessary to further study the swirling characteristic of natural gas under supersonic flow conditions. In this paper, a swirling device is newly designed 
and installed in the upstream of the converging part of a Laval nozzle. The swirling characteristics of the natural gas flow is analyzed in detail using CFD simulations, while the effect of the structure of the swirling device on the swirling flow is also discussed in the newly designed supersonic swirling separator.

\section{Supersonic separators}

For a supersonic separator, there are two different methods to design and install the swirling device. One is that the swirling device is designed as a delta wing and installed after the nozzle exit. The other is that a set of static guide blades is designed as the swirling device and located in the upstream of the nozzle entrance. For the first method, the swirling flow is generated in the supersonic velocity, which causes some complex shock waves and increases the flow resistance. Consequently, the second approach is employed here to design a new swirling device for our supersonic separator.

In this kind of supersonic separator, on one hand, the existence of the swirling flow may damage the expansion effect of the Laval nozzle. On the other hand, the swirling device also produces some resistance when natural gas flows through it. It means that we not only enable the swirling device to produce a large centrifugal field to separate the water and heavy hydrocarbons, but also need to control the swirling flow to prevent the damage of the expansion characteristics of the Laval nozzle. Depending on this requirement, the swirling device is designed as a set of helical blades located on the surface of an ellipsoid body, as shown in Fig. 1. The whole length of the designed supersonic separator is $1140.50 \mathrm{~mm}$, involving the nozzle converging part of $149.00 \mathrm{~mm}$, the diverging part of $306.60 \mathrm{~mm}$, and the diffuser section of $384.90 \mathrm{~mm}$. The diameters for the separator inlet and outlet are $80.00 \mathrm{~mm}$ and $40.00 \mathrm{~mm}$, respectively. The diameters of the outer wall and inner body at the 
nozzle throat are $20.00 \mathrm{~mm}$ and $13.96 \mathrm{~mm}$, respectively. The diameter of the inner body at the nozzle outlet is $6.42 \mathrm{~mm}$.

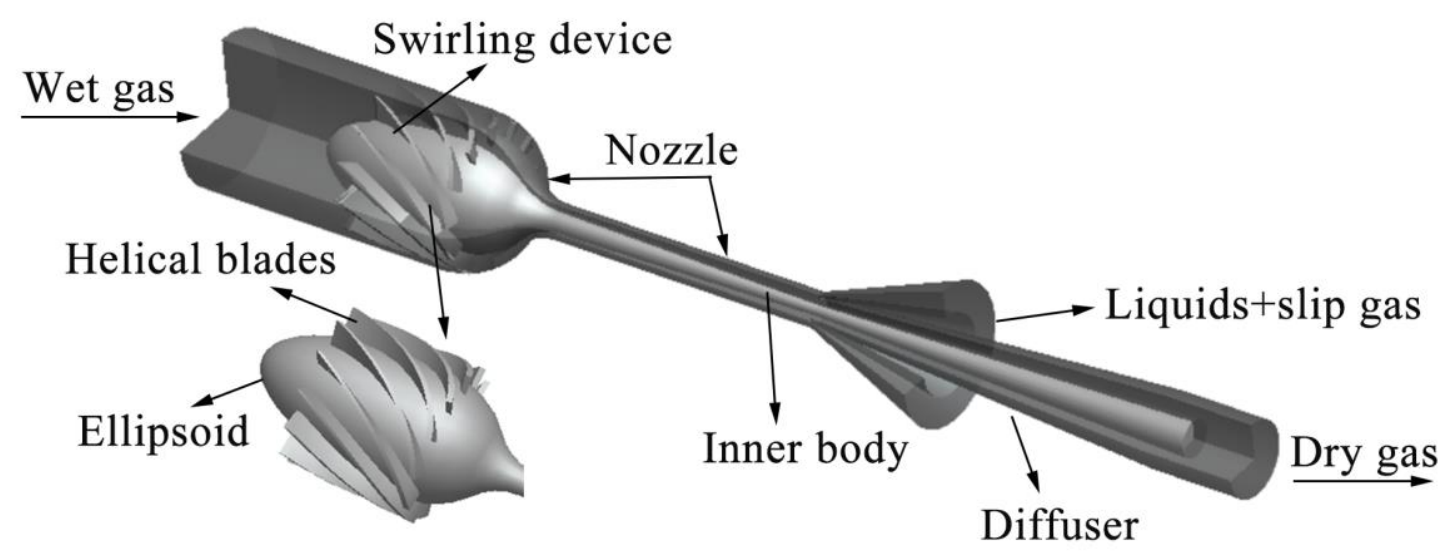

Fig.1 Structure of a supersonic separator and swirling device

\section{Mathematical Model}

\subsection{Governing Equations}

The governing equations describing the natural gas flow in a supersonic separator involve the continuity, momentum and energy equations, and their general forms can be described as:

Continuity equation:

$$
\frac{\partial \rho}{\partial t}+\nabla\left(\cdot \rho \boldsymbol{u}_{i}\right)=(
$$

Momentum equation:

$$
\frac{\partial}{\partial t}\left(\rho \boldsymbol{u}_{i}+\nabla \cdot\left(\rho \boldsymbol{u} \boldsymbol{u}_{j}\right) \nexists p+\nabla \cdot \overline{\bar{\tau}}(+\phi g g+\boldsymbol{F}\right.
$$

where $\rho g$ and $\boldsymbol{F}$ are the gravitational body force and external body forces. $\overline{\bar{\tau}}$ is the stress tensor and can be given by

$$
\overline{\bar{\tau}}=\mu\left[\left(\nabla \boldsymbol{u}+\nabla \boldsymbol{u}^{T}\right)-\frac{2}{3} \nabla \cdot(\boldsymbol{u} \overline{\bar{I}})\right]
$$

where $\overline{\bar{I}}$ is the unit tensor.

Energy equation: 


$$
\frac{\partial}{\partial t}(\rho E)+\nabla \cdot(\rho \boldsymbol{u} E)=-p \nabla \cdot \boldsymbol{u}-\nabla \cdot q+\nabla \cdot(\boldsymbol{u} \overline{\bar{\tau}})
$$

where $E$ is the total energy; $q$ is the heat flux.

\subsection{Turbulence model}

It is particularly important to employ an appropriate turbulence model for the numerical calculation of fluid flow in a supersonic separator, in which the anisotropic effect is significant due to the turbulence flow in a high speed with a strong swirl. In the current turbulence models, one of the greatest advantages of the Reynolds Stress Model is that it can accurately calculate the anisotropic effect, such as the buoyancy effect, rotating effect, near-wall effect, etc. Therefore, we employ the Reynolds Stress Model [22] to model the complex swirling flow in a supersonic separator. The transport equations for the transport of the Reynolds stresses may be written as follows:

$$
\begin{aligned}
& \frac{\partial}{\partial t}\left(\rho \overline{\boldsymbol{u}_{i}^{\prime} \boldsymbol{u}_{j}^{\prime}}\right)+\frac{\partial}{\partial x_{k}}\left(\rho \boldsymbol{u}_{\boldsymbol{k}} \overline{\boldsymbol{u}_{i}^{\prime} \boldsymbol{u}_{j}^{\prime}}\right)=-\frac{\partial}{\partial x_{k}}\left(\rho \overline{\boldsymbol{u}_{i}^{\prime} \boldsymbol{u}_{j}^{\prime} \boldsymbol{u}_{k}^{\prime}}+\overline{p\left(\delta_{k j} \boldsymbol{u}_{i}^{\prime}+\delta_{i k} \boldsymbol{u}_{j}^{\prime}\right)}\right) \\
& +D_{L, i j}+P_{i j}+G_{i j}+\phi_{i j}+\varepsilon_{i j}+F_{i j}+S_{r}
\end{aligned}
$$

where $D_{L, i j}, P_{i j}, G_{i j}, \phi_{i j}, \varepsilon_{i j}, F_{i j}$ and $S_{r}$ present the molecular diffusion, stress production, buoyancy production, pressure strain, dissipation, system rotation, source term. Please refer to ref. [23] for a detailed description of the RSM model.

The turbulent kinetic energy, $k$, and the turbulent dissipation rate, $\varepsilon$, are solved by the following equations, respectively:

$$
\begin{array}{r}
\frac{\partial}{\partial t}(\rho k)+\frac{\partial}{\partial x_{i}}(\rho k \boldsymbol{u})=\frac{\partial}{\partial x_{j}}\left[\left(\mu+\frac{\mu_{t}}{\sigma_{k}}\right) \frac{\partial k}{\partial x_{j}}\right]+\frac{1}{2}\left(P_{i i}+G_{i i}\right)-\rho \varepsilon\left(1+2 M_{t}^{2}\right)+S_{k} \\
\frac{\partial}{\partial t}(\rho \varepsilon)+\frac{\partial}{\partial x_{i}}(\rho \varepsilon \boldsymbol{u})=\frac{\partial}{\partial x_{j}}\left[\left(\mu+\frac{\mu_{t}}{\sigma_{\varepsilon}}\right) \frac{\partial \varepsilon}{\partial x_{j}}\right]+C_{\varepsilon 1} \frac{1}{2}\left[P_{i i}+C_{\varepsilon 3} G_{i i}\right] \frac{\varepsilon}{k}-C_{\varepsilon 2} \rho \frac{\varepsilon^{2}}{k}+S_{\varepsilon}
\end{array}
$$

where: 


$$
\mu_{t}=\rho C_{\mu} \frac{k^{2}}{\varepsilon}
$$

where $C_{1 \varepsilon}=1.44, C_{\varepsilon 2}=1.92, \sigma_{k}=0.82, \sigma_{\varepsilon}=1.0$.

3.3 Numerical schemes

The detailed methods for our numerical simulation are listed in Table 1, including the discretization schemes for governing equation, pressure-velocity coupling method, boundary conditions, etc.

Table1 Numerical methods for the CFD modeling

\begin{tabular}{ll}
\hline Name & Methods \\
\hline Discretization schemes for governing & Finite Volume Method \\
equation & \\
Pressure-velocity coupling & SIMPLE algorithm [24] \\
Turbulence model & Reynolds Stress Model \\
Boundary conditions & Inlet: Pressure inlet \\
& Outlet: Pressure outlet \\
& Solid wall: Adiabatic and No slip \\
Near-wall & Non-equilibrium wall functions \\
Convergence condition & Energy equation: below $10^{-6}$ \\
(Residual value) & Other equations: below $10^{-3}$ \\
& Total mass error in the inlet/outlet mass flow \\
& rates: below $10^{-4}$. \\
\hline
\end{tabular}

\section{Results and discussion}

\subsection{Validation and verification of numerical method}

In this paper, the numerical simulation is carried out with our experimental separator 
[25] by using the above mentioned numerical methods. The comparison between the numerical and experimental results is shown in Fig. 2. It can be seen that the predicted results are less than the experimental values under conditions of the strong swirling flows. For example, the mass flow rate error reaches 3.6\%, when the swirl strength, $S$, is about 0.906 , which is defined as the ratio between the tangential, $v_{t}$, and critical velocity, $a_{c}$, at the nozzle throat, $S=v_{t} / a_{c}$. The difference is caused by the neglect of the wall friction in the numerical calculations, which exaggerates the effect of swirling flow on the mass flow rate. In general, the numerical results agree well with the experimental data, demonstrating that the CFD modeling can accurately predict the strong swirling flow in a supersonic separator.

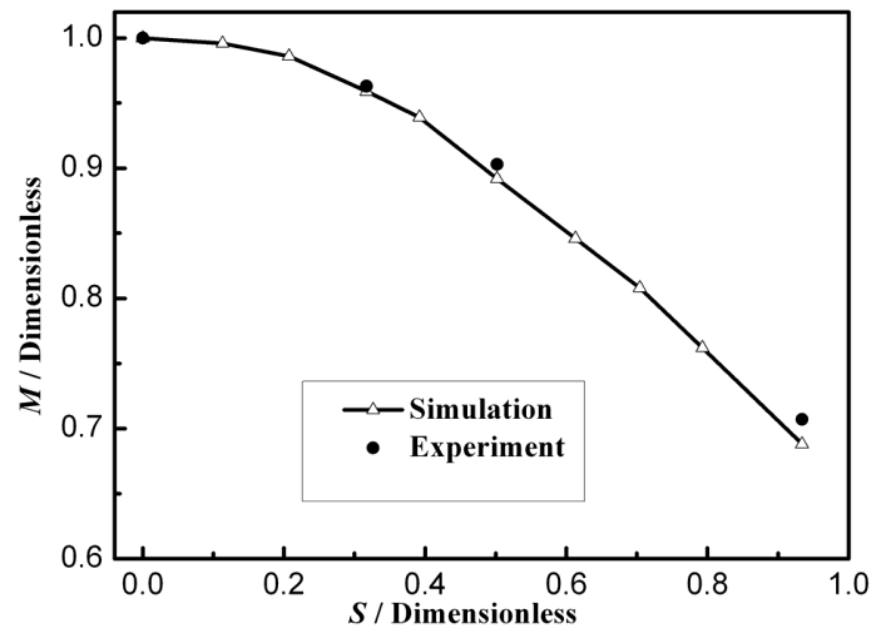

Fig.2 Effect of swirl strength on mass flow rates in a supersonic separator

The grid quality and density can greatly affect the accuracy and reliability of the numerical calculations. In our simulation cases, the swirling device is meshed with unstructured grid for its complex geometry, while the structured grids are generated for all other parts. The grid dependence analysis is performed to test the effect of the grid density on the numerical results. Three grid numbers are adopted for the sensitivity analysis including the coarse (282 643), medium (638 357), and fine (1 436 168) grids. The flow conditions for this simulation include the inlet gas pressure, flow 
rate and temperature, which are $4 \mathrm{MPa}, 1000000 \mathrm{Nm}^{3} / \mathrm{d}$, and $303.15 \mathrm{~K}$, respectively. The Mach numbers in the diverging part of the annular nozzle are depicted in Fig. 3. The gas Mach number at the nozzle throat are shown in Table 2. The Mach number with the coarse grid significantly deviates from the other two cases. The relative error between the medium and fine grids is less than 3\%. Therefore, the quantity of grid is about 600,000 for our numerical calculation in consideration of the computing accuracy and efficiency.

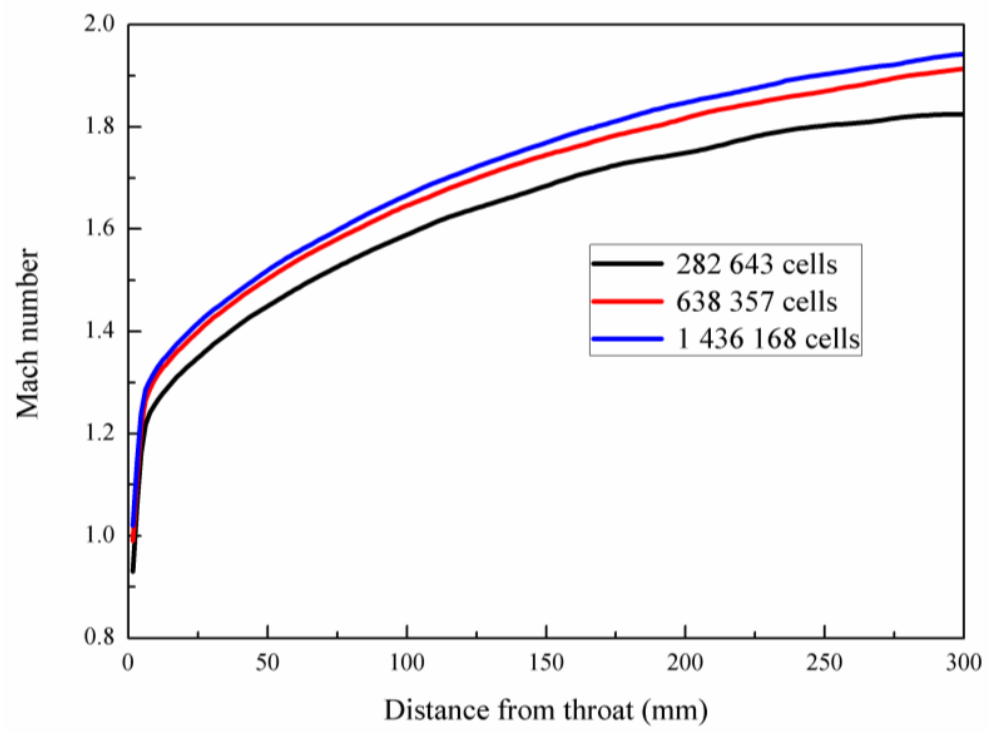

Fig.3 Gas Mach number in the diverging part of the annular nozzle

Table 2. Mach number at nozzle throat with different cell numbers

\begin{tabular}{|c|c|c|c|}
\hline Cell numbers & $\begin{array}{l}\text { Mass flow rate } \\
(\mathrm{kg} / \mathrm{s})\end{array}$ & $\begin{array}{l}\text { Mach number } \\
\text { at nozzle throat }\end{array}$ & Error $(\%)$ \\
\hline 282643 & 7.8446 & 0.93 & 8.82 \\
\hline 638357 & 7.8210 & 0.99 & 2.94 \\
\hline 1436168 & 7.7993 & 1.02 & 0 \\
\hline
\end{tabular}

\subsection{Supersonic swirling flow in an annular nozzle}

In a supersonic separator, natural gas is accelerated to a supersonic speed when it flows through the diverging part of a Laval nozzle, leading to the low pressure and 
temperature condition. It causes that the water vapor and heavy hydrocarbons condense into liquid droplets, which can be separated under the influence of the centrifugal forces. For this reason, it is particularly necessary to investigate the swirling characteristics of natural gas flow in the diverging part of a Laval nozzle.

Figs. 4 and 5 describe the axial and tangential velocity of natural gas flow at the throat, middle and outlet cross-section of an annular Laval nozzle. We can see that the gas axial velocity distributes uniformly in the central area of the annular channel, while significant velocity gradients are observed in the near-wall region. The axial velocity is approximately $500 \mathrm{~m} / \mathrm{s}$ near the outer wall at the nozzle outlet. Consequently, the condensed droplets can be carried to the liquid collection space and be separated from the liquid outlet.

The tangential velocity presents an uneven distribution along the radial direction at three representative cross-sections. But the maximum tangential velocity does not appear in the central area of the annular flow channel. On the contrary, the maximum value appears in the region closer to the inner wall surface due to the strong swirls, approximately $20 \%$ of the cross-section radius of the annular flow channel. For example, the maximum tangential velocity is about $150 \mathrm{~m} / \mathrm{s}$ at the annular nozzle outlet. Correspondingly, the maximum centrifugal acceleration reaches 4500000 $\mathrm{m} / \mathrm{s}^{2}$, which is estimated by $a=v_{t}^{2} / r$, where $a$ is the centrifugal acceleration, $v_{t}$ is tangential velocity, $r$ is radius.

The gas static pressure and temperature at three respective cross-sections are shown in Figs. 6 and 7. It can be seen that the static pressure reaches a maximum at the outer wall surface and the minimum at the inner surface on account of the existence of the strong swirls. It indicates that there is a significant radial pressure gradient in the cyclone separation process. The gas static temperature is sufficiently 
low in the central area of the annular channel, about $-70{ }^{\circ} \mathrm{C}$ at the nozzle outlet, which creates a suitable condition for the condensation of water and heavy hydrocarbon components.

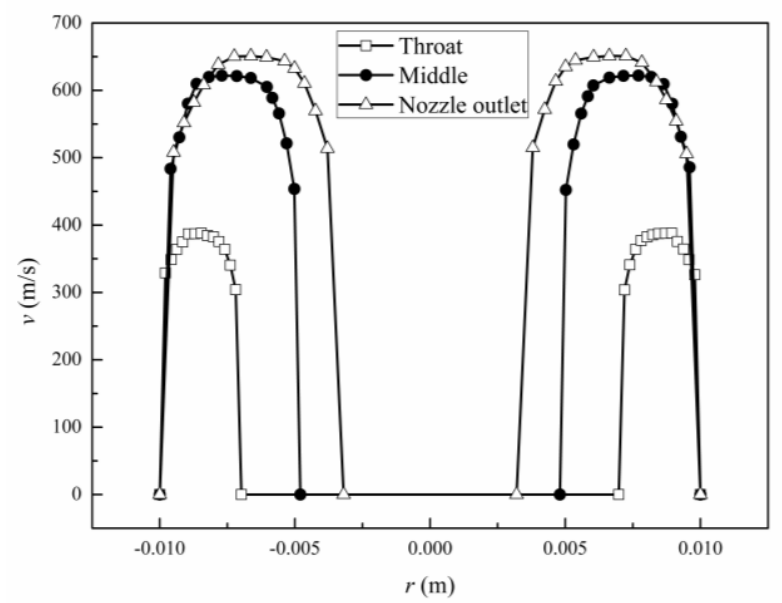

Fig.4 Gas axial velocity at three representative cross-sections

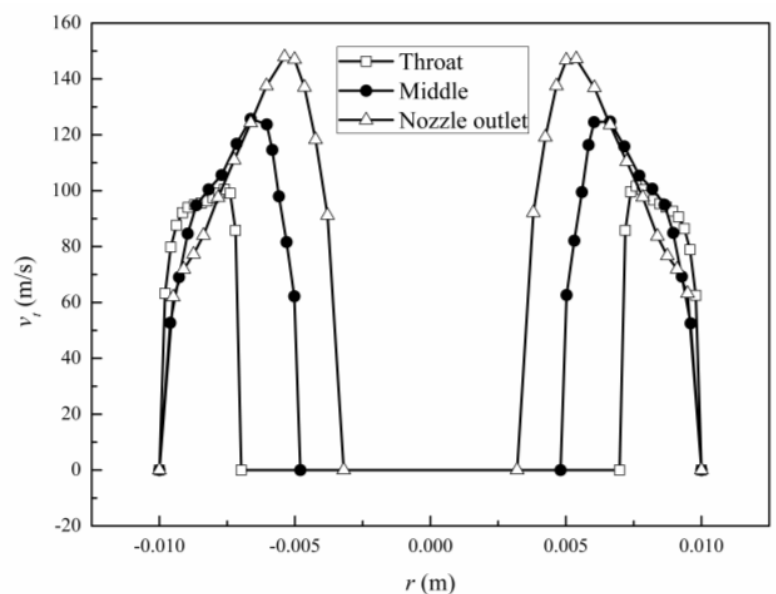

Fig.5 Gas tangential velocity at representative cross-sections 

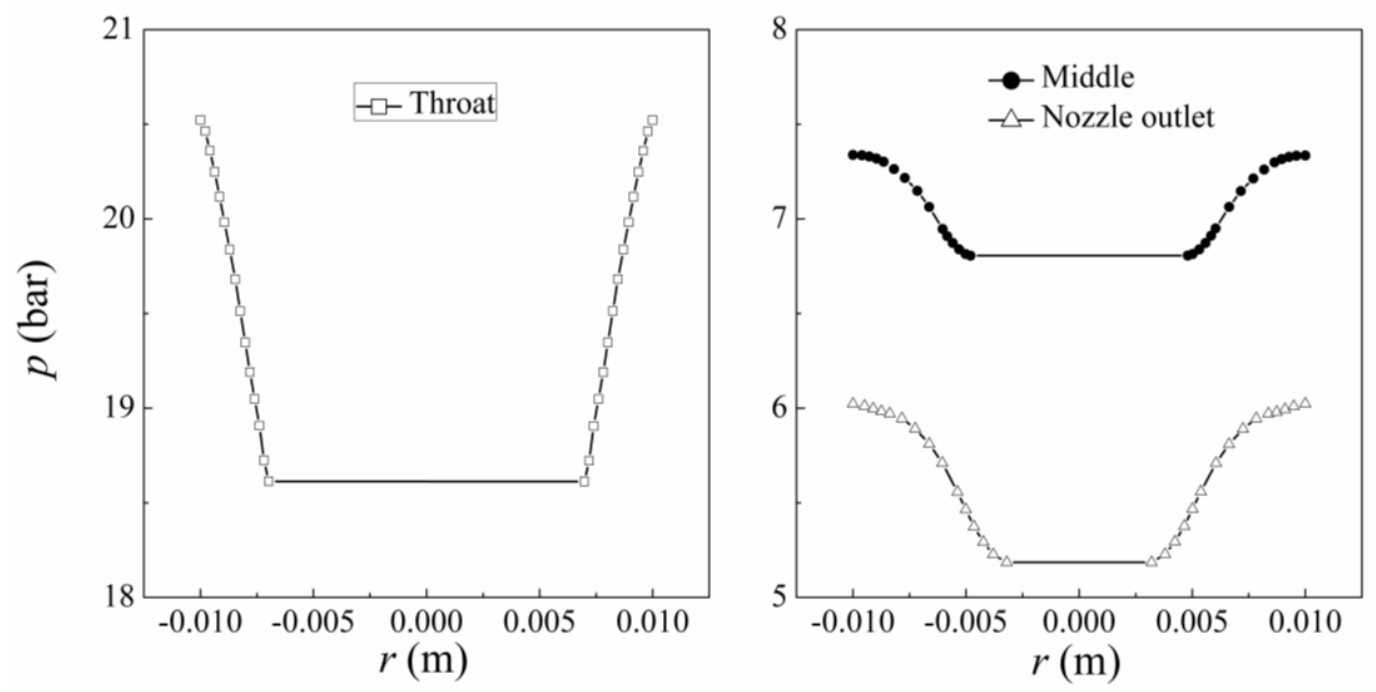

Fig.6 Static pressure at representative cross-sections

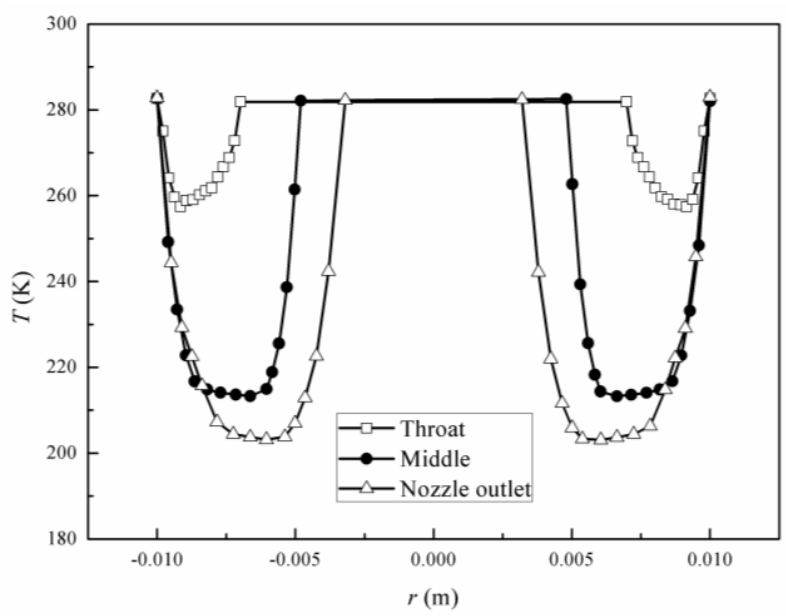

Fig.7 Static temperature at representative cross-sections

\subsection{Effect of blade angles}

The blade angle is defined as the acute angle between the outlet angle of the helical blade and the axial direction. From the design perspective of a swirling device, the blade angle is a key factor to generate a centrifugal force. The influence of different blade angles on the swirling flow in the annular Laval nozzle is studied numerically considering angles of $10.2^{\circ}, 19.7^{\circ}, 30.0^{\circ}, 40.4^{\circ}, 50.1^{\circ}, 60.0^{\circ}, 70.4^{\circ}$ and $79.8^{\circ}$, respectively.

Fig. 8 depicts the gas static temperature and tangential velocity at the designed 
annular nozzle outlet under various blade angles. The tangential velocity significantly increases with the increase of the blade angles. It indicates that a strong swirling flow can be generated in a large blade angle to separate the condensed droplets from gas liquid mixtures. But meanwhile, the increasing blade angle also results in the rise of the gas static temperature, which means that the low pressure and temperature condition is weakened as a result of the swirling flow. That is, the swirling characteristic and expansion effect are mutually exclusive. For instance, when the blade angle is less than $40^{\circ}$, the maximum tangential velocity is only about $100 \mathrm{~m} / \mathrm{s}$, indicating a poor swirling effect, although the gas static temperature reaches $-80{ }^{\circ} \mathrm{C}$ at this condition. Likewise, the minimum static temperature increases to $-30{ }^{\circ} \mathrm{C}$, showing a weak expansion effect, although the maximum tangential velocity reaches $270 \mathrm{~m} / \mathrm{s}$, if the blade angle is up to $79.8^{\circ}$. Therefore, we need to balance the swirling characteristic and expansion effect in the design of a supersonic separator, in which the swirling device is installed in the entrance of a Laval nozzle. Correspondingly, the blade angle of $45-70^{\circ}$ is recommended for the design of a swirling device.

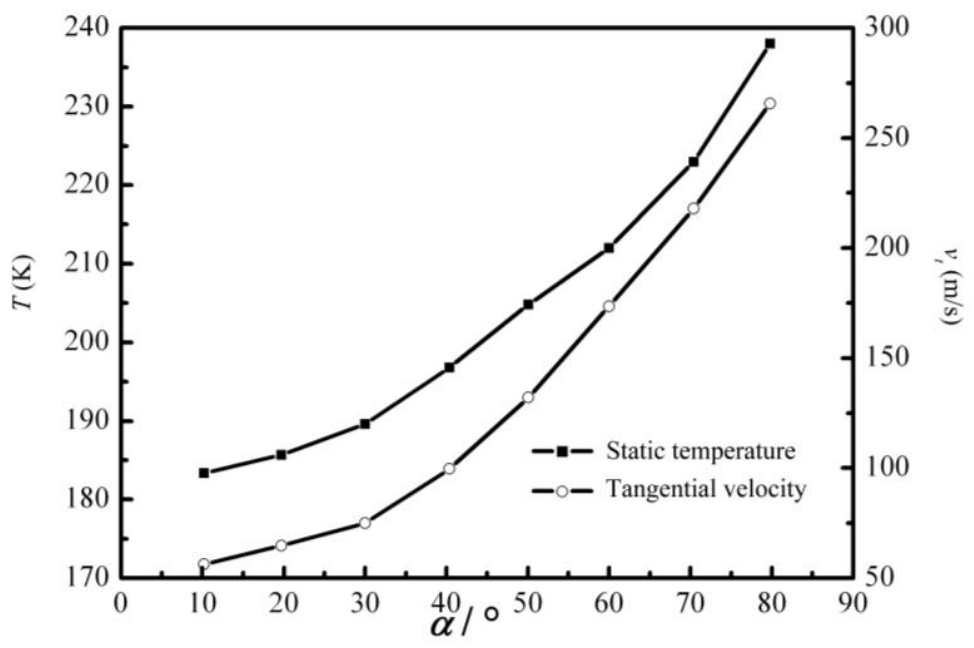

Fig.8 Effect of blade angles on gas swirling characteristics

\subsection{Effect of blade numbers}

Fig. 9 shows the gas static pressure and tangential velocity at the annular nozzle 
outlet with different blade numbers. The tangential velocity increases from $105 \mathrm{~m} / \mathrm{s}$ to $200 \mathrm{~m} / \mathrm{s}$ with the increase of the blade number, $n_{v}$, with a corresponding centrifugal acceleration of $2000000 \mathrm{~m} / \mathrm{s}^{2}$ and $7600000 \mathrm{~m} / \mathrm{s}^{2}$, meaning that the swirling flow is enhanced. Simultaneously, the gas static temperature increases from $-87{ }^{\circ} \mathrm{C}$ to $-62{ }^{\circ} \mathrm{C}$, reducing the expansion effect of the annular nozzle. However, the impact of the blade numbers on the swirling characteristic and expansion effect gradually declines, when it is more than 4 blades. For example, the tangential velocity increases from $165 \mathrm{~m} / \mathrm{s}$ to $200 \mathrm{~m} / \mathrm{s}$, and the gas static temperature rises from $-70{ }^{\circ} \mathrm{C}$ to $-62{ }^{\circ} \mathrm{C}$, while the blade numbers increases from 4 to 12 . Hence, the $4-8$ helical blades can be used for a swirling device to balance the swirling characteristic and expansion effect in a supersonic separator.

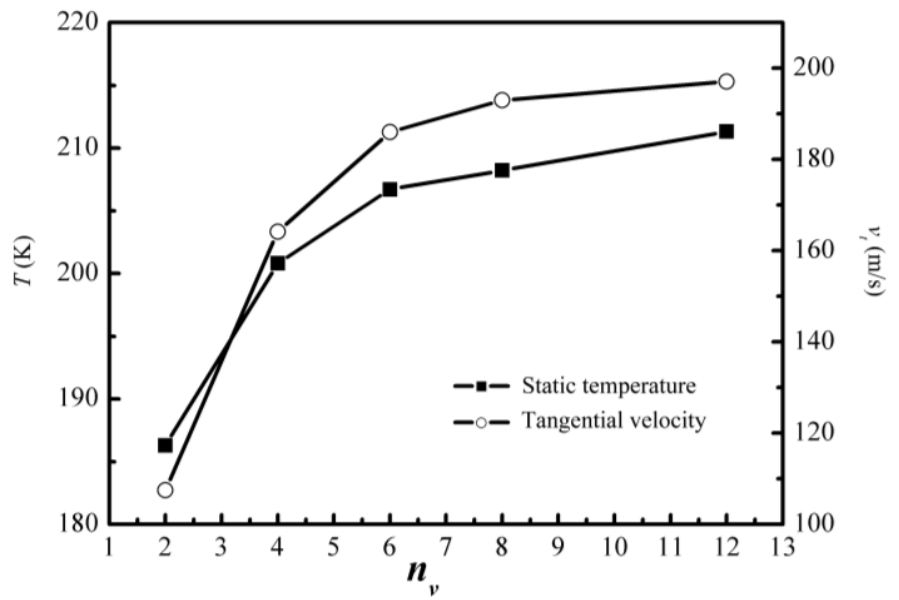

Fig. 9 Effect of blade numbers on gas swirling characteristics

\section{Conclusions}

The ellipsoid and helical blades are newly designed as a swirling device, which is installed in the entrance of an annular nozzle for a supersonic separator. This kind of swirling device not only generates a strong swirling motion, but also reduces the flow resistance. The mathematical model is developed to allow detailed studies of the swirling characteristic of the natural gas flow through the annular channel at a 
supersonic speed. We find that the gas velocity, static pressure and temperature distributes non-uniformly in the radial direction as a result of the viscous heating and strong swirling flow. The maximum tangential velocity is closer to the inner wall surface, and the maximum centrifugal acceleration can reach $4300000 \mathrm{~m} / \mathrm{s}^{2}$, which provides a sufficient centrifugal field to remove the condensed droplets from gas liquid mixtures. If $4-8$ blades with the swirling angle of $40-70^{\circ}$ are used in the swirling device, the strong swirling flow and low temperature can be obtained in the newly designed supersonic separator.

\section{Acknowledgements}

This work was supported in part by the Natural Science Foundation of Jiangsu Province, China (No. BK20150270), the General Program of Natural Science Research Project of Jiangsu Province Universities and Colleges (No. 15KJB440001), and the Scientific Research Project of Changzhou (No. CJ20140044, CJ20159034). C. Wen acknowledges the support of the H.C. Ørsted fellowship co-funded by Marie Curie Actions at the Technical University of Denmark, DTU.

\section{Nomenclature}

$\begin{array}{ll}a & \text { centrifugal acceleration } \\ a_{c} & \text { critical velocity } \\ C_{\varepsilon 1} & \text { constant } \\ C_{\varepsilon 2} & \text { constant } \\ C_{\varepsilon 3} & \text { constant } \\ D_{L, i j} & \text { molecular diffusion } \\ E & \text { total energy } \\ \boldsymbol{F} & \text { external body forces } \\ F_{i j} & \text { production by system rotation }\end{array}$


acceleration of gravity

$G_{i j} \quad$ buoyancy production

$\overline{\bar{I}} \quad$ unit tensor

$k \quad$ turbulent kinetic energy

$M_{t} \quad$ turbulent Mach number

$p \quad$ pressure

$P_{i j} \quad$ stress production

$q \quad$ heat flux

$r \quad$ radius

S $\quad$ swirl strength

$S_{k} \quad$ source term

$S_{r} \quad$ source term

$S_{\varepsilon} \quad$ source term

$t \quad$ time

$\boldsymbol{u} \quad$ velocity

$v_{t} \quad$ tangential velocity

$\delta_{i j} \quad$ Kronecker delta

$\varepsilon \quad$ turbulent dissipation rate

$\varepsilon_{i j} \quad$ turbulent dissipation

$\mu \quad$ gas viscosity

$\mu_{t} \quad$ turbulent viscosity

$\rho \quad$ gas density

$\sigma_{k} \quad$ constant

$\sigma_{\varepsilon} \quad$ constant

$\overline{\bar{\tau}} \quad$ stress tensor 
$\phi_{i j} \quad$ pressure strain

\section{References}

[1] T. Prenzel, T.L.M. Guedes, F. Schlüter, M. Wilhelm, K. Rezwan, Tailoring surfaces of hybrid ceramics for gas adsorption-From alkanes to $\mathrm{CO}_{2}$, Sep. Purif. Technol. 129 (2014) 80-89.

[2] Y.E. Kim, S.J. Moon, Y.I. Yoon, S.K. Jeong, K.T. Park, S.T. Bae, S.C. Nam, Heat of absorption and absorption capacity of $\mathrm{CO}_{2}$ in aqueous solutions of amine containing multiple amino groups, Sep. Purif. Technol. 122 (2014) 112-118.

[3] H. N.A. Halim, A.M. Shariff, M.A. Bustam, High pressure $\mathrm{CO}_{2}$ absorption from natural gas using piperazine promoted 2-amino-2-methyl-1-propanol in a packed absorption column, Sep. Purif. Technol. 152 (2015) 87-93.

[4] W. Ye, J. Lin, H.T. Madsen, E.G. Søgaard, C. Hélix-Nielsen, P. Luis, B. Van der Bruggen, Enhanced performance of a biomimetic membrane for $\mathrm{Na}_{2} \mathrm{CO}_{3}$ crystallization in the scenario of $\mathrm{CO}_{2}$ capture, J. Membr. Sci. 498 (2016) 75-85.

[5] W. Ye, J. Lin, J. Shen, P. Luis, B. Van der Bruggen, Membrane crystallization of sodium carbonate for carbon dioxide recovery: Effect of impurities on the crystal morphology, Cryst. Growth Des. 13 (2013) 2362-2372.

[6] V. Alfyorov, L. Bagirov, L. Dmitriev, V. Feygin, S. Imayev, J.R. Lacey, Supersonic nozzle efficiently separates natural gas components, Oil Gas J. 103 (2005) 53-58.

[7] P.B. Machado, J.G. Monteiro, J.L. Medeiros, H.D. Epsom, O.Q. Araujo, Supersonic separation in onshore natural gas dew point plant, J. Nat. Gas Sci. Eng. 6 (2012) 43-49.

[8] E. Jassim, M.A. Abdi, Y. Muzychka, Computational fluid dynamics study for flow of natural gas through high-pressure supersonic nozzles: part 1. real gas 
effects and shockwave, Pet. Sci. Technol. 26 (2008) 1757-1772.

[9] E. Jassim, M.A. Abdi, Y. Muzychka, Computational fluid dynamics study for flow of natural gas through high-pressure supersonic nozzles: part 2. nozzle geometry and vorticity, Pet. Sci. Technol. 26 (2008) 1773-1785.

[10]A. Karimi, M.A. Abdi, Selective dehydration of high-pressure natural gas using supersonic nozzles. Chem. Eng. Process. 48 (2009) 560-568.

[11]M. Malyshkina, The structure of gas dynamic flow in a supersonic separator of natural gas, High Temp. 46 (2008) 69-76.

[12]M. Malyshkina, The procedure for investigation of the efficiency of purification of natural gases in a supersonic separator, High Temp. 48 (2010) 244-250.

[13] Y. Yang, C. Wen, S. Wang, Y. Feng, Theoretical and numerical analysis on pressure recovery of supersonic separators for natural gas dehydration, Appl. Energy 132 (2014) 248-253.

[14]Y. Yang, C. Wen, S. Wang, Y. Feng, Numerical simulation of real gas flows in natural gas supersonic separation processing, J. Nat. Gas Sci. Eng. 21 829-836.

[15]B.M. Vaziri, A. Shahsavand, Optimal selection of supersonic separators inlet velocity components via maximization of swirl strength and centrifugal acceleration, Sep. Sci. Technol. 50 (2015) 752-759.

[16]Q.F. Ma, D.P. Hu, J.Z. Jiang, Z.H. Qiu, A turbulent Eulerian multi-fluid model for homogeneous nucleation of water vapour in transonic flow, Int. J. Comput. Fluid Dynamics 23 (2009) 221-231.

[17]Q.F. Ma, D.P. Hu, J.Z. Jiang, Z.H. Qiu, Numerical study of the spontaneous nucleation of self-rotational moist gas in a converging-diverging nozzle, Int. J. Comput. Fluid Dynamics 24 (2010) 29-36. 
[18] S.H.R. Shooshtari, A.Shahsavand, Reliable prediction of condensation rates for purification of natural gas via supersonic separators, Sep. Purif. Technol. 116 (2013) 458-470.

[19]S.H.R. Shooshtari, A.Shahsavand, Predictions of wet natural gases condensation rates via multi-component and multi-phase simulation of supersonic separators, Korean J. Chem. Eng. 31 (2014) 1845-1858.

[20]M. Castier, Modeling and simulation of supersonic gas separations, J. Nat. Gas Sci. Eng. 18 (2014) 304-311.

[21] M. Haghighi, K.A. Hawboldt, M. A.Abdi, Supersonic gas separators: Review of latest developments, J. Nat. Gas Sci. Eng. 27 (2015) 109-121.

[22] S. Pope, Turbulent Flows. Cambridge University Press, Cambridge, 2000

[23] ANSYS Fluent User Manual, 2014, ANSYS INC.

[24] S.V. Patankar, D.B. Spalding, A calculation procedure for heat, mass and momentum transfer in three-dimensional parabolic flows, Int. J. Heat Mass Tran. 15 (1972) 1787-1806.

[25]C. Wen, X. Cao, Y. Yang, J. Zhang, Swirling effects on the performance of supersonic separators for natural gas separation, Chem. Eng. Technol. 34 (2011) 1575-1580. 